\title{
Feline Lower Urinary Tract Disease (Flutd) - An Emerging Problem of Recent Era
}

\author{
Tariq $\mathrm{A}^{* 1}$, Rafique $\mathrm{R}^{2}$, Abbas $\mathrm{SY}^{2}$, Khan $\mathrm{MN}^{2}$, Huma $\mathrm{I}^{2}$, Perveen $\mathrm{S}^{2}$ and Kamran $\mathrm{M}^{1}$ \\ ${ }^{1}$ Internee at Veterinary Research Institute (VRI), Lahore, Punjab, Pakistan \\ ${ }^{2}$ Veterinary Research Institute (VRI), Lahore, Punjab, Pakistan
}

*Corresponding author: Tariq A, Internee at Veterinary Research Institute (VRI), Faculty of Veterinary Sciences (FVS), University of Agriculture Faisalabad (UAF), Punjab, Pakistan, E-mail: dr.arslantariq3418@ live.com

Citation: Tariq A, Rafique R, Abbas SY, Khan MN, Huma I, et al. (2014) Feline Lower Urinary Tract Disease (Flutd) - An Emerging Problem of Recent Era. J Vet Sci Anim Husb 2(3): 302. doi: 10.15744/2348-9790.1.503

Received Date: August 21, 2014 Accepted Date: October 22, 2014 Published Date: October 24, 2014

\begin{abstract}
Feline lower urinary tract disease (FLUTD) is a supposed to a life threatening condition in cats, especially in males (Toms), when obstructive. Early diagnosis and treatment is necessary otherwise it may lead to death. Condition appears with stranguria, pollakiuria, dysuria and sometime in severe conditions hematuria and anuria may be present. Similar five cases of age ranging from 3-6 years, with common history of commercial feed and indoor placement were examined during the course of 3 month. Firstly clinical evaluation including clinical parameters and physical manipulation was done followed by laboratory tests. Complete blood count (CBC) didn't give any significant change but urinalysis results were quite doubtful with high values of specific gravity (SP), $\mathrm{pH}$, erythrocyte, bacterial and leukocyte count. Urine culture reveled $E$. coli infection which is found to be sensitive to different antibiotics in culture sensitivity test. Multiple treatments were done for fourteen days including flushing with normal saline, specific with antibiotics (ciprofloxacin), Non-steroidal anti-inflamatory (ketoprofen) and supportive with infusion (Normal saline) which end up with successful recovery within 2 weeks. Fortnightly visits were made for six months to check for recurrence but didn't find any sign yet.
\end{abstract}

Keywords: Toms; Urinalysis; Feline lower urinary tract disease (FLUTD); Ciprofloxacin

\section{Introduction}

Cats usually suffer through a common urinary tract infection called "Feline Lower urinary tract infection (FLUTD)" which occurs in in both genders equally [1]. Hostutler et al. [2] claimed its first accurate description in 1925 but still some authors didn't recognize this as a disease but only a condition [3,4]. FLUTD usually appears in 2-6 year age with 1.5-8\% prevalence while very rarely under 1 year or 10 year of age $[2,4]$. Condition may present with anorexia, fever, stranguria, dysuria, periuria and pollakiuria and microscopic hematuria but in severe cases macroscopic hematuria and anuria may be present due to complete obstruction of urethra [2,3]. Commonly UTI infection has bacterial origin like E. coli, Enterococcus spp and Staphylococcus felis and Corynebacterium urealyticum infection [4,5]. As disease is considered as life threatening so study has impact to highlight important aspects of disease so that early diagnosis and treatment may possible after understanding the clinical aspects in future.

\section{Main Body}

Total five no. of male Persian cats (Toms) named (Tom I-V) visited veterinary teaching hospital at University of Agriculture Faisalabad (UAF) during the course of 3 months from November, 2013 to January 2014, with chief complaint of anorexia, stranguria, lethargic and depressed behavior. Investigation exposed a common history of indoor placement on litter and fed on commercial feed. Age of the Tom I and III were approx. 3 year old while remaining were approx. 5-6 year old. After attainment of comprehensive history, clinical parameters like, temperature, pulse, respiration and dehydration were recorded as given below (Table 1). All toms were presenting stranguria, prominent pollakiuria in tom I-IV while tom-V presented with anuria. Macroscopic hematuria was also recorded in tom-III and V. Distended urinary bladder was felt when manipulated physically. Stones were drawn from urethra as stone are known be the FLUTD in cats previously described by [6], which come up as struvite when evaluated from diagnostic lab. Blood sample was collection directly from vein by sterile syringe while urine samples were drawn by cystocentesis by a sterile and evauated by using "Hematology Analyzer" and "dip-stick" respectively. Blood profile didn't give any significant change but only a minute elevation in neutrophils as mention in Table 2, also described by [4,6]. On other end urinalysis explored overall increase $\mathrm{pH}$, specific gravity (SP), erythrocyte and leukocyte along with bacterial [7] (Table 3).

Culture of urine samples on MacKoncky agar was come up with growth of E coli in all cases which then pass through culture sensitivity test as previously performed by Srivastava and Syed [1]. Growth show sensitivity three positive (+++) against Levofloxacin, enrofloxacin and Ciprofloxacin while give two positive (++) against Amoxicillin and Kanamycin. The condition was diagnosed as Feline lower urinary tract disease (FLUTD) with urinary infection on evidence of clinical signs and laboratory results. 


\begin{tabular}{|c|c|c|c|c|c|c|c|}
\hline Parameters & Units & Tom-I & Tom-II & Tom-III & Tom-IV & Tom-V & $\begin{array}{c}\text { Standard } \\
\text { ranges }\end{array}$ \\
\hline \multicolumn{8}{|c|}{ Clinical parameters documented on zero day } \\
\hline Temperature & ${ }^{\circ} \mathbf{F}$ & 104.4 & 104.2 & 104.6 & 104.2 & 104.8 & $100.5-102.5$ \\
\hline Respiration & Beats/min & 42 & 37 & 44 & 39 & 47 & $16-40$ \\
\hline Heart rate & Beats/min & 138 & 147 & 159 & 141 & 156 & $120-140$ \\
\hline Dehydration & $\%$ & 5 & 5 & 7 & 4 & 6 & Nil \\
\hline \multicolumn{8}{|c|}{ Results documented after consecutive treatment of 7 days } \\
\hline Temperature & ${ }^{\circ} \mathbf{F}$ & 102.2 & 102.0 & 103.0 & 102.0 & 102.8 & $100.5-102.5$ \\
\hline Respiration & Beats/min & 37 & 35 & 42 & 21 & 38 & $16-40$ \\
\hline Heart rate & Beats/min & 133 & 129 & 145 & 123 & 136 & $120-140$ \\
\hline Dehydration & $\%$ & Nil & Nil & 2 & Nil & 1 & Nil \\
\hline \multicolumn{8}{|c|}{ Results documented after consecutive treatment of 14 days } \\
\hline Temperature & ${ }^{\circ} \mathbf{F}$ & 101.6 & 101.2 & 102.2 & 101.8 & 102.0 & $100.5-102.5$ \\
\hline Respiration & Beats/min & 33 & 32 & 27 & 35 & 28 & $16-40$ \\
\hline Heart rate & Beats/min & 135 & 133 & 128 & 129 & 126 & $120-140$ \\
\hline Dehydration & $\%$ & Nil & Nil & Nil & Nil & Nil & Nil \\
\hline
\end{tabular}

${ }^{*}$ References ranges: The Merck Manual, Small Animal Clinical Diagnosis by Laboratory Methods

Table 1: Documentation of clinical parameters on different intervals

\begin{tabular}{|c|c|c|c|c|c|c|c|}
\hline Parameters & Units & Tom-I & Tom-II & Tom-III & Tom-IV & Tom-V & $\begin{array}{c}\text { Standard } \\
\text { ranges }\end{array}$ \\
\hline \multicolumn{8}{|c|}{ Results documented on day of $\mathbf{1}^{\text {st }}$ visit (zero day) } \\
\hline RBC & $10^{6} / \mu \mathrm{l}$ & 7.9 & 8.2 & 6.6 & 9.0 & 7.2 & $5.0-10.0$ \\
\hline PCV & $\%$ & 29 & 27 & 35 & 27 & 33 & $24-34$ \\
\hline Hbg conc. & $\mathrm{g} / \mathrm{dl}$ & 12.3 & 12.8 & 11.9 & 13.1 & 10.7 & $10-15$ \\
\hline WBCs & $10^{3} / \mu \mathrm{l}$ & 14.3 & 16.5 & 17.2 & 12.1 & 16.8 & 5.5-19.5 \\
\hline Platelets & $10^{3} / \mu \mathrm{l}$ & 587 & 665 & 473 & 603 & 503 & $300-800$ \\
\hline Neutrophils & $\%$ & 69 & 67 & 71 & 65 & 68 & $45-64$ \\
\hline Lymphocytes & $\%$ & 29 & 30 & 27 & 32 & 31 & $27-36$ \\
\hline Monocytes & $\%$ & 1 & 2 & 1 & 2 & 1 & $0-5$ \\
\hline Eosinophil's & $\%$ & 1 & Nil & 1 & 1 & Nil & $0-4$ \\
\hline Basophils & $\%$ & Nil & 1 & Nil & Nil & Nil & $0-1$ \\
\hline \multicolumn{8}{|c|}{ Results documented after consecutive treatment of 14 days } \\
\hline $\mathrm{RBC}$ & $10^{6} / \mu \mathrm{l}$ & 6.7 & 5.8 & 7.3 & 7.6 & 8.7 & $5.0-10.0$ \\
\hline PCV & $\%$ & 30 & 28 & 28 & 29 & 32 & $24-34$ \\
\hline Hbg conc. & $\mathrm{g} / \mathrm{dl}$ & 13.3 & 11.7 & 12.5 & 14.3 & 13.1 & $10-15$ \\
\hline WBCs & $10^{3} / \mu \mathrm{l}$ & 16.3 & 13.4 & 14.2 & 14.8 & 17.8 & 5.5-19.5 \\
\hline Platelets & $10^{3} / \mu \mathrm{l}$ & 623 & 713 & 599 & 646 & 619 & $300-800$ \\
\hline Neutrophils & $\%$ & 58 & 62 & 55 & 63 & 57 & $45-64$ \\
\hline Lymphocytes & $\%$ & 35 & 32 & 36 & 29 & 34 & $27-36$ \\
\hline Monocytes & $\%$ & 3 & 4 & 5 & 5 & 4 & $0-5$ \\
\hline Eosinophil's & $\%$ & 3 & 2 & 3 & 3 & 4 & $0-4$ \\
\hline Basophils & $\%$ & Nil & Nil & 1 & Nil & 1 & $0-1$ \\
\hline
\end{tabular}

${ }^{*}$ References ranges: The Merck Manual, Small Animal Clinical Diagnosis by Laboratory Methods

Table 2: Documentation of complete blood count (CBC) on different intervals 


\begin{tabular}{|c|c|c|c|c|c|c|}
\hline \multicolumn{7}{|c|}{ Results documented on day of 1st visit (zero day) } \\
\hline Parameters & Tom-I & Tom-II & Tom-III & Tom-IV & Tom-V & $\begin{array}{l}\text { Standard } \\
\text { ranges }\end{array}$ \\
\hline Color & Yellowish & Yellowish & Reddish yellow & Yellowish & Reddish yellow & Amber yellow \\
\hline Specific gravity & 1.055 & 1.055 & 1.060 & 1.050 & 1.050 & $1.015-1.050$ \\
\hline $\mathrm{pH}$ & 7.8 & 7.68 & 8.1 & 7.9 & 8.0 & $5.5-7$ \\
\hline Bilirubin & -ve & -ve & -ve & -ve & -ve & Nil \\
\hline Glucose (g/dl) & -ve & -ve & -ve & -ve & -ve & Nil \\
\hline Ketones & -ve & -ve & -ve & -ve & -ve & Nil \\
\hline Erythrocytes/HPF & 2 & 1 & $4+$ & 2 & $3+$ & Nil \\
\hline Bacteria/CFU & 2 & -ve & 2 & 1 & 2 & Nil \\
\hline \multicolumn{7}{|c|}{ Results documented after consecutive treatment of 14 days } \\
\hline Color & Yellowish & Yellowish & Radish yellow & Yellowish & Radish yellow & Amber yellow \\
\hline Specific gravity & 1.025 & 1.030 & 1.030 & 1.035 & 1.025 & $1.015-1.050$ \\
\hline $\mathrm{pH}$ & 5.9 & 6.6 & 6.9 & 7.0 & 5.8 & $5.5-7$ \\
\hline Bilirubin & -ve & -ve & -ve & -ve & -ve & Nil \\
\hline Glucose (g/dl) & -ve & -ve & -ve & -ve & -ve & Nil \\
\hline Ketones & -ve & -ve & -ve & -ve & -ve & Nil \\
\hline Erythrocytes/HPF & -ve & -ve & 1 & -ve & -ve & Nil \\
\hline Bacteria/CFU & -ve & -ve & -ve & -ve & -ve & Nil \\
\hline
\end{tabular}

*References ranges: The Merck Manual, Small Animal Clinical Diagnosis by Laboratory Methods

Table 3: Documentation of urinalysis on different intervals

Regarding treatment a muscular relaxant Diazepam @ $0.2 \mathrm{mg} / \mathrm{kg}$ was injected intramuscularly at the time of arrival just to relax the animals and urinary bladder was flushed with Normal saline by using 4 french size naso-gastric tube as previously performed by Zohaib et al., [4]. Ciprofloxacin @ 8mg/kg intramuscularly and ketoprofin @ 1mg/kg b.w intramuscularly was recommended for 5 days whereas supportive therapy with normal saline @ $150 \mathrm{ml} /$ day for first 7 days and $75 \mathrm{ml} /$ days for up-to 14 days intravenously was given by taking in consideration of results of Zohaib et al., [4]. Owners were advised to stop the usage of dry feed instead of that homemade feed (chicken soup and boiled milk) and provision of fresh water ad libidum was recommended. All animals were announced to be recovered in 14 days on evidence of urinalysis and clinical parameters within normal ranges by collecting and analyzing samples similar techniques as previously used. Animal were revisited fortnightly for six month to grasp reappearance but were found perfectly alright.

\section{Discussion}

Feline Lower urinary tract disease (FLUTD) is common condition of cats kept indoors, and fed on dry diet with less water availability are more likely to have this disease as recorded in respected study. Recently body weight is also considered as a major risk factor $[2,8]$. In respected report all presented toms were straining and crying during act of urination while two of them presenting hematuria and anuria which are considered as main presenting signs of disease according to [3,4]. FLUTD and urolithiasis are present respectively in 55-69\% and 13 to $28 \%$ cases while urethral obstruction and urinary tract infections are uncommon and just participated 10 to $21 \%$ and 1 to $8 \%$ cases respectively.

Non-obstructive disease appears at equal ratio in both sexes but obstructive frequently appears in males and about $18-58 \%$ of cat population [2,4] as appeared only in males in respected report. On an average out of $20 \%$ case of urinary calculi $80 \%$ are struvite of which commercial feed is a main cause are dissolve within 36 days if concurrent infection does not occur [4]. In case of concurrent infection may takes avg. 44 days to dissolve [2,4]. FLUTD was diagnosed on evidence of urinalysis, urine culture and complete blood count (CBC) which revealed high values of urinalysis and E. coli infection in urine culture. Stones collected from urethra were found to be as struvite which are present in $80 \%$ cases described by Zohaib et al., [4]. After recognition of infection culture sensitivity test was performed which give positive results for ciprofloxacin as by [2] and treatment was started with ciprofloxacin and ketoprofen.

According to [2] disease signs normally takes a week to be resolve but if concurrent infection is there then antibiotics are recommended for 7 days so ciprofloxacin for one weeks was recommended as E. coli infection was present. According to earlier studies [2] obstructive and non-obstructive disease chances of recurrence are $45 \%$ and 39\% within 6 month and 1 year respectively which explain that still there is a room for improvement. So all animals were check fortnightly for 6 month for re-emergence of condition but still didn't find any signs of disease. 


\section{Conclusion}

In conclusion, disease is found to have a significant factor of dry feed and constrict indoor housing. This communication reveled elevated level of urinalysis values ( $\mathrm{pH}$, Specific gravity, erythrocyte and leukocyte count) with E.coli infection, however with no significant change in blood profile was seen. All animals respond well against treatment and diet change plan.

\section{References}

1. Srivastava AK, Syed B (2012) Management of urinary tract infection in cats. Indian Journal of Canine Practice 4: 94-5.

2. Hostutler RA, Chew DJ, DiBartola SP (2005) Recent Concepts in Feline Lower Urinary Tract Disease. Vet Clin North Am Small Anim Practe 35: 147-70.

3. Bailiff NL, Westropp JL, Nelson RW, Sykes JE, Owens SD, et al. (2008) Evaluation of urine specific gravity and urine sediment as risk factors for urinary tract infections in cats. Vet Clin Pathol 37: 317-22.

4. Zohaib A, Taj Z, Sial AUR, Naeem MA, Saqlein M (2013) Feline lower urinary tract disease-report of four cases. Pakistan Vet J 33: $131-2$.

5. Cavana P, Zanatta R, Nebbia P, Miniscalco B, Vittone V, et al. (2008) Corynebacterium urealyticum urinary tract infection in a cat with urethral obstruction. J Feline Med Surg 10: 269-73.

6. Syme HM (2012) Stones in cats and dogs: What can be learnt from them? Arab J Urology 10: 230-9.

7. Tvedten H, Willard M (2004) Small Animal Clinical Diagnosis by Laboratory Methods. (4 ${ }^{\text {th }}$ edn), Saunders Publishers, St Louis, Missouri, USA.

8. Kerr KR (2013) Companion Animals Symposium: dietary management of feline lower urinary tract symptoms. J Anim Sci 91: $2965-75$.

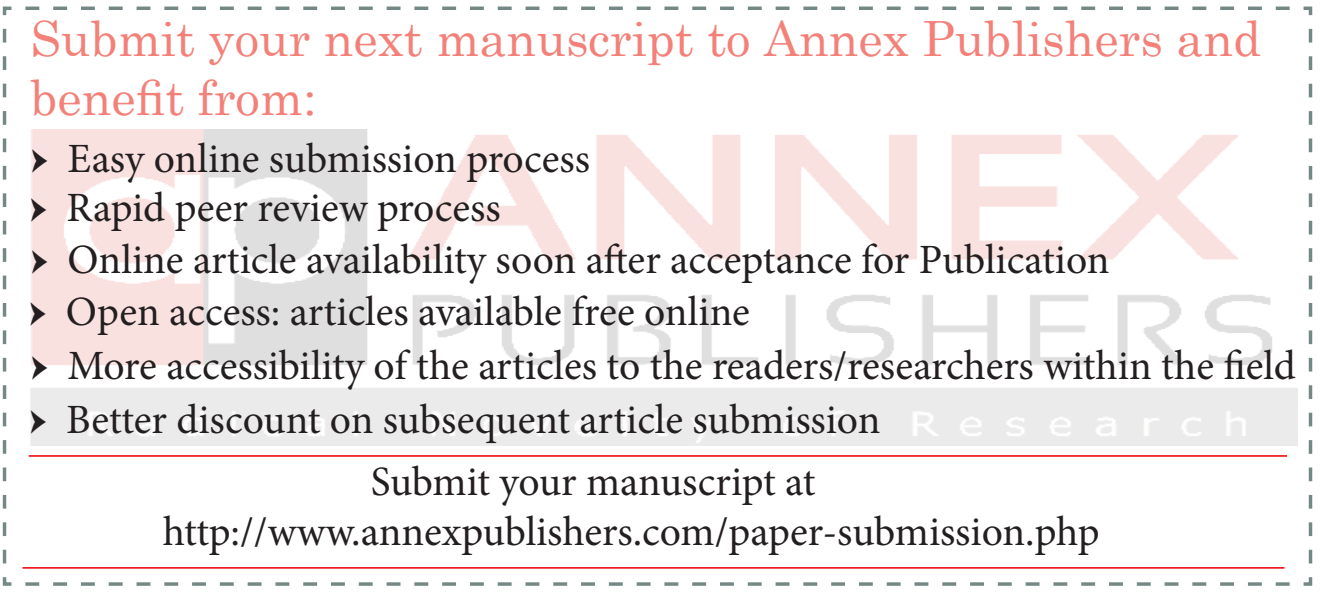

